\title{
Reinforcement learning, Sequential Monte Carlo and the EM algorithm
}

\author{
VIVEK S BORKAR ${ }^{1, *}$ and ANKUSH V JAIN ${ }^{1,2}$ \\ ${ }^{1}$ Department of Electrical Engineering, Indian Institute of Technology Bombay, Powai, Mumbai 400076, India \\ ${ }^{2}$ Present Address: Graviton Research Capital LLP, 14th Floor, Tower C, Building 8, Cyber City, Gurugram, \\ Haryana 122002, India \\ e-mail: borkar.vs@gmail.com; jainankush2308@gmail.com
}

MS received 9 February 2017; revised 19 November 2017; accepted 19 November 2017; published online 29 June 2018

\begin{abstract}
Using the expression for the unnormalized nonlinear filter for a hidden Markov model, we develop a dynamic-programming-like backward recursion for the filter. This is combined with some ideas from reinforcement learning and a conditional version of importance sampling in order to develop a scheme based on stochastic approximation for estimating the desired conditional expectation. This is then extended to a smoothing problem. Applying these ideas to the EM algorithm, a reinforcement learning scheme is developed for estimating the partially observed log-likelihood function. A stochastic approximation scheme maximizes this function over the unknown parameter. The two procedures are performed on two different time scales, emulating the alternating 'expectation' and 'maximization' operations of the EM algorithm. We also extend this to a continuous state space problem. Numerical results are presented in support of our schemes.
\end{abstract}

Keywords. Nonlinear filtering and smoothing; Sequential Monte Carlo; reinforcement learning; importance sampling; EM algorithm.

\section{Introduction}

The problem of estimating the state of a hidden Markov model (HMM) at a given time, given the observations up to that time, is solved 'in principle' by a nonlinear filter. The latter exploits Bayes rule to give a recursive scheme for computing the conditional law of the current state given current history of observations [2]. This, however, is often computationally intractable. This has led to the development of Sequential Monte Carlo (SMC) or the related particle filters, which use Markov Chain Monte Carlo (MCMC) techniques to simulate realizations of the state process conditioned on observations, and estimate the desired quantities by averaging as in the classical MCMC [2]. This, again, inherits the usual issues concerning MCMC, such as possibly high variance [3]. Recently, reinforcement learning has been used as an alternative to pure MCMC, the idea being to combine MCMC with

Work of VSB supported in part by a J C Bose Fellowship and a grant for 'Distributed Computation for Optimization over Large Networks and High Dimensional Data Analysis' from the Department of Science and Technology, Government of India. A part of this work was presented at the Workshop on Information Theory and Applications, San Diego, CA, February 2014 [1].

*For correspondence classical numerical schemes so as to retain some advantages of both [4]. As argued in [4], it inherits from MCMC lower per iterate computation and memory requirement than those of the deterministic numerical schemes. From numerical schemes, it inherits a lower variance than that of pure MCMC. The latter, as pointed out in ibid., is because the learning schemes, through 'one step' analysis, estimate one step conditional expectations rather than the full expectations. In a sense one can think of this as RaoBlackwellization of MCMC.

The latter development, however, has been for fully observed Markov processes. Our aim here is to extend these ideas to HMMs. Putting the filter for HMMs in a form suitable for reinforcement learning, however, turns out to be a bit tricky. One has to fall back upon the so called unnormalized filter, originally due to Zakai in continuous time and space case [5]. We use the discrete time and space version developed in [6] and Chapter VIII of [7] (see [8] for a comprehensive treatment). Writing a backward recursion for the unnormalized quantities that is akin to a dynamic programming recursion, we incorporate therein a conditional version of importance sampling introduced in [9]. This enables us to render the computation amenable to a reinforcement learning scheme. This is our first important contribution, which also sets this work apart from the classical 'backward simulation' methods [10]. Our scheme 
- replaces the conditional average in the backwarddynamic-programming-like recursion by an actual simulated value according to the said conditional law, weighted appropriately by a 'conditional likelihood ratio' to account for our choice of the sampling measure, and

- makes an incremental correction using stochastic approximation, which does the averaging. (Details follow.)

The entire set-up is then extended to the 'smoothing' problem of estimating a function of the state at a certain time given observations both past and some way into the future (rather, estimate conditional law of a past quantity given current history of observations). This sets up the stage for our objective of applying these ideas to the Expectation-Maximization (EM) algorithm. The smoothing problem turns out to be a little more involved than the pure filtering problem. We also sketch extensions to general state and observation spaces. This build-up leads to our main aim, dealt with in section 3, viz., to apply this methodology to the celebrated EM algorithm for parameter estimation in HMMs. Note that what we thus have is an offline batch mode algorithm for EM with lower variance. The aforementioned results on filtering are steps towards this goal; the algorithms are not best suited for filtering applications, which are usually on-line and recursive.

An excellent and comprehensive treatment of existing techniques for inference in HMMs can be found in [11].

\section{A reinforcement learning approach to filtering}

\subsection{The algorithm}

Consider the HMM given by the (state, observation) processes $\left(X_{n}, Y_{n}\right), n \geq 0$, taking values in a finite product space $\mathcal{S} \times \mathcal{O}$, such that

$$
P\left(X_{n+1}=i, Y_{n+1}=j \mid X_{m}, Y_{m}, m \leq n\right)=p\left(i, j \mid X_{n}\right)
$$

for a prescribed transition probability function $p(\cdot, \cdot \cdot \cdot)$. Let $\pi_{n}:=$ the conditional distribution of $X_{n}$ given $\mathcal{F}_{n}:=\sigma\left(Y_{i}, i \leq n\right), n \geq 0$. We assume $\pi_{0}$ to be known. Given $N>1$, our aim is to estimate

$$
\pi_{N} f:=\sum_{i \in \mathcal{S}} \pi_{N}(i) f(i)=E\left[f\left(X_{N}\right) \mid Y_{m}, m \leq N\right]
$$

for a prescribed $f: \mathcal{S} \mapsto \mathcal{R}$, based on observations $Y_{m}, 1 \leq m \leq N . \quad$ Let $\quad P(y):=[[p(j, y \mid i)]]_{i, j \in S}$, $\mathbf{1}:=[\mathbf{1}, \mathbf{1}, \cdots, \mathbf{1}]^{\mathbf{T}}$. Note that $P(y)$ is in general a substochastic matrix. Then $\pi_{n}$ written as a row vector is given by the nonlinear filter

$$
\pi_{n+1}=\frac{\pi_{n} P\left(Y_{n+1}\right)}{\pi_{n} P\left(Y_{n+1}\right) \mathbf{1}}=\frac{v_{n+1}}{v_{n+1} \mathbf{1}}
$$

where the unnormalized conditional law $v_{n}$, also written as a row vector, is given by the linear iterates

$$
v_{n+1}=v_{n} P\left(Y_{n+1}\right), v_{0}=\pi_{0} .
$$

Thus, to evaluate $\pi_{n} f$, we can evaluate

$$
\frac{v_{n} f}{v_{n} \mathbf{1}}
$$

Hence, we need a general scheme to evaluate $v_{N} f$ for a generic $f: \mathcal{S} \mapsto \mathcal{R}$. Define

$$
V_{n}(i)=v_{N} f \text { when } v_{n}(\cdot)=\delta_{i}, \cdot, n \leq N,
$$

where $\delta_{\text {., }}$ is the Kronecker delta. (That is, $v_{N} f$ is computed as if the state was known to be exactly $i$ at time $n$.) Then it is easy to see that $V$ is completely characterized by the 'backward equation' with an associated terminal condition:

$$
\begin{gathered}
V_{n}(i)=\sum_{j} p\left(j, Y_{n+1} \mid i\right) V_{n+1}(j), n<N, \\
V_{N}(i)=f(i) .
\end{gathered}
$$

The backward equation and the terminal condition for evaluating $v_{n} \mathbf{1}$ are

$$
\begin{gathered}
\hat{V}_{n}(i)=\sum_{j} p\left(j, Y_{n+1} \mid i\right) \hat{V}_{n+1}(j), n<N, \\
\hat{V}_{N}(i)=1 .
\end{gathered}
$$

Rewrite Eq. (1) as

$$
V_{n}(i)=\sum_{j} q(j \mid i)\left(\frac{p\left(j, Y_{n+1} \mid i\right)}{q(j \mid i)}\right) V_{n+1}(j), n<N,
$$

where $Q:=[[q(j \mid i)]]_{i, j \in \mathcal{S}}$ is an irreducible stochastic matrix. We can view (5) as a one-step analysis or 'dynamic programming' recursion for evaluating the finite horizon multiplicative (or 'risk-sensitive') cost

$$
E\left[\prod_{n=0}^{N-1}\left(\frac{p\left(X_{n+1}, Y_{n+1} \mid X_{n}\right)}{q\left(X_{n+1} \mid X_{n}\right)}\right) f\left(X_{N}\right)\right] .
$$

Consider a Markov chain $\left\{\breve{X}_{n}\right\}$ with transition matrix $Q$ and initial distribution $\pi_{0}$. The law of this chain will be our 'importance sampling' measure. Simulate $M \gg 1$ independent runs $\left\{\breve{X}_{m}^{k}, 0 \leq m \leq N\right\}, 1 \leq k \leq M$, of the chain with initial distribution $\pi_{0}$.

For comparison purposes, also consider a naive MCMCbased filter to estimate $v_{N} f$, which would be as follows. Define the quantities

$$
\begin{aligned}
\bar{\gamma}_{0}^{M} & :=\frac{1}{M} \sum_{k=1}^{M} \prod_{n=0}^{N-1}\left(\frac{p\left(\breve{X}_{n+1}^{k}, Y_{n+1} \mid \breve{X}_{n}^{k}\right)}{q\left(\breve{X}_{n+1}^{k} \mid \breve{X}_{n}^{k}\right)}\right) f\left(\breve{X}_{N}^{k}\right), \\
\check{\gamma}_{0}^{M} & :=\frac{1}{M} \sum_{k=1}^{M} \prod_{n=0}^{N-1}\left(\frac{p\left(\breve{X}_{n+1}^{k}, Y_{n+1} \mid \breve{X}_{n}^{k}\right)}{q\left(\breve{X}_{n+1}^{k} \mid \breve{X}_{n}^{k}\right)}\right) .
\end{aligned}
$$


These are nothing but sample averages of the summands over independent simulation runs, amenable to "strong law of large numbers' as we shall soon see. The summands in turn are samples of the quantity of interest (e.g., $f\left(X_{N}\right)$ in the first case) multiplied by the likelihood function to correct for the aforementioned importance sampling measure. $\frac{\gamma_{0}^{M}}{\gamma_{0}^{M}}$ is an estimate of $\pi_{N} f$ for large $M$. This follows from the following theorem.

Theorem $1 \quad\left(\bar{\gamma}_{n}^{M}, \check{\gamma}_{0}^{M}\right) \stackrel{M \uparrow \infty}{\rightarrow}\left(v_{N} f, v_{N} \mathbf{1}\right)$ almost surely (a.s.)

Proof This is immediate from the strong law of large numbers.

The alternative we propose is a 'stochastic approximation' or 'reinforcement learning' scheme given by

$$
\begin{aligned}
\tilde{V}_{N}^{k+1}(i)= & f(i), \\
\tilde{V}_{n}^{k+1}(i)= & \left(1-a(k) I\left\{\breve{X}_{n}^{k+1}=i\right\}\right) \tilde{V}_{n}^{k}(i) \\
& +a(k) I\left\{\breve{X}_{n}^{k+1}=i\right\} \\
& {\left[\left(\frac{p\left(\breve{X}_{n+1}^{k+1}, Y_{n+1} \mid i\right)}{q\left(\breve{X}_{n+1}^{k+1} \mid i\right)}\right) \tilde{V}_{n+1}^{k+1}\left(\breve{X}_{n+1}^{k+1}\right)\right], n<N . } \\
\check{V}_{N}^{k+1}(i)=1 & , \\
\check{V}_{n}^{k+1}(i)= & \left(1-a(k) I\left\{\breve{X}_{n}^{k+1}=i\right\}\right) \check{V}_{n}^{k}(i) \\
& +a(k) I\left\{\breve{X}_{n}^{k+1}=i\right\} \\
& {\left[\left(\frac{p\left(\breve{X}_{n+1}^{k+1}, Y_{n+1} \mid i\right)}{q\left(\breve{X}_{n+1}^{k+1} \mid i\right)}\right) \check{V}_{n+1}^{k+1}\left(\breve{X}_{n+1}^{k+1}\right)\right], n<N, }
\end{aligned}
$$

where $\{a(k)\}$ is a sequence of positive step-sizes satisfying the standard conditions $\sum_{k} a(k)=\infty, \sum_{k} a(k)^{2}<\infty$.

The way to arrive at this, e.g., the first set of equations, is to treat (5) as a dynamic programming equation for the 'value function' $\left\{V_{n}\right\}$ with the term in parentheses on the right hand side as the multiplicative 'running cost' (cf. (6)), and invoke the corresponding reinforcement learning scheme for approximate dynamic programming. Equation (5) is a backward recursion. To obtain the reinforcement learning scheme, one first replaces the conditional expectation on the right hand side by an actual evaluation at a transition executed according to the conditional distribution in question, yielding the quantity in large square brackets on the right in (7)-(8). We then make an incremental move towards it. That is, we take the next iterate as a convex combination of the current iterate and the aforementioned quantity, with a slowly decreasing weight $a(k)$ for the latter. This weight is the wellknown slowly decreasing step-size schedule of stochastic approximation, which does the averaging for you. Since our computations use a simulation run (from 'time' 0 to $N$ ) of a Markov chain with transition probabilities $q(\cdot \cdot \cdot)$, the $i^{\text {th }}$ component of the estimate for value function $V_{n}$ at time $n$ can be updated only if the chain is in state $i$ at (simulated) time $n$.
This is because this is the only situation when one sees a transition out of $i$ at time $n$. This is the reason for having the indicator $I\left\{\breve{X}_{n}^{k+1}=i\right\}$ on the right hand side, which ensures that only this and no other component is updated. In particular, it makes this an asynchronous stochastic approximation. We analyse its convergence properties simply by invoking the by-now standard 'o.d.e.' approach to the analysis of stochastic approximation, leading to

Theorem $2 \quad \tilde{V}_{n}^{M} \stackrel{M \uparrow \infty}{\rightarrow} V_{n}, \check{V}_{n}^{M} \stackrel{M \uparrow \infty}{\rightarrow} \hat{V}_{n} \forall n$, a.s.

Proof Almost-sure boundedness of the iterates is clear. Let $\eta(n, i):=$ the a.s. limit of $\frac{1}{M} \sum_{k=0}^{M} I\left\{\breve{X}_{n}^{k}=i\right\}$ as $M \uparrow \infty$, which exists by the strong law of large numbers. By standard 'o.d.e.' analysis of stochastic approximation algorithms (see Chapter 6 of [12]), it suffices to consider the 'o.d.e. limit' of the aforementioned iteration. This is given by

$$
\begin{aligned}
& \frac{d}{d t} v_{n, i}=\eta(n, i)\left(\sum_{j} p\left(j, Y_{n+1} \mid i\right) v_{n+1, j}(t)-v_{n, i}(t)\right), \\
& \frac{d}{d t} v_{n, i}^{\prime}=\eta(n, i)\left(\sum_{j} p\left(j, Y_{n+1} \mid i\right) v_{n+1, j}^{\prime}(t)-v_{n, i}(t)\right),
\end{aligned}
$$

for $0 \leq n<N, i \in \mathcal{S}$, with $v_{N, i}(t) \equiv f(i), v_{N, i}^{\prime}(t) \equiv 1$. The presence of the $\eta \mathrm{s}$ is the effect of asynchrony. Using a backward induction on $n$, one can show that these o.d.e.s converge to the unique solutions $V, \hat{V}$ of (1)-(2) and (3)(4), respectively as $t \uparrow \infty$. The claim then follows by Corollary 4, p. 18 of [12].

Remark We mention in passing an 'adaptive importance sampling' variant, though we do not pursue it much here. (We do, however, include some relevant numerical experiments later.) The aforementioned scheme, where we used a fixed sampling kernel $q$, may be termed as a 'naive' importance sampling scheme. In adaptive importance sampling as introduced in [9], we replace $q\left(\breve{X}_{n+1}^{k+1} \mid i\right)$ by a time-dependent transition kernel

$$
q_{n}^{k+1}(j \mid i):=\frac{\tilde{p}^{k+1}\left(j, Y_{n+1} \mid i\right)}{\sum_{u^{\prime}} \tilde{p}^{k+1}\left(u^{\prime}, Y_{n+1} \mid i\right)},
$$

where for a prescribed $0<\epsilon<<1$

$$
\tilde{p}^{k+1}\left(u^{\prime}, y^{\prime} \mid u\right):=\max \left(p\left(u^{\prime}, y^{\prime} \mid u\right)\left(\frac{\tilde{V}_{n+1}^{k}\left(u^{\prime}\right)}{\tilde{V}_{n}^{k}(u)}\right), \epsilon\right) .
$$

$\left\{\breve{X}_{n}^{k+1}\right\}$ are correspondingly generated according to these transition probabilities. The justification for this scheme is as follows:

$$
q_{n}^{*}(x \mid z):=p\left(x, Y_{n+1} \mid z\right)\left(\frac{V_{n+1}(x)}{V_{n}(z)}\right)
$$

is seen to be the ideal 'zero variance' choice of measure for importance sampling, except that its definition involves the 
very quantities $\left\{V_{n}\right\}$ that we want to estimate. Thus we replace $V_{n}$ by its estimate to obtain an approximation to the zero variance measure. The parameter $\epsilon$ avoids division by zero or a very small number in early stages of the iteration. See [9] for a more detailed discussion of relevant issues. Note also that the $q^{k}$ s for (3) will be different: their definition has $\hat{V}_{n}$ in place of $V_{n}$.

\subsection{Extensions}

1. Smoothing: The aforementioned development is intended as a step towards our first generalization, viz., to smoothing, which is a key step towards building a reinforcement learning scheme for the EM algorithm. The smoothing problem is to estimate

$$
E\left[f\left(X_{n}\right) \mid Y_{m}, m \leq N\right]
$$

for $N>n$. A simple application of the Bayes rule shows that this equals

$$
\begin{aligned}
& \frac{\sum_{x_{0}, \cdots, x_{N}} \pi_{0}\left(x_{0}\right)\left(\prod_{k=0}^{N-1} p\left(x_{k+1}, Y_{k+1} \mid x_{k}\right)\right) f\left(x_{n}\right)}{\sum_{x_{0}, \cdots, x_{N}} \pi_{0}\left(x_{0}\right)\left(\prod_{k=0}^{N-1} p\left(x_{k+1}, Y_{k+1} \mid x_{k}\right)\right)} \\
& =\frac{v_{n}\left(f \hat{V}_{n}\right)}{v_{N} \mathbf{1}}
\end{aligned}
$$

where $f \hat{V}_{n}$ denotes the componentwise product. The important difference with the filtering problem is the presence of the term $\hat{V}_{n}$ in the numerator accounting for the observations after time $n$, which carry additional information about the state at time $n$. By proceeding analogously to the preceding section, the reinforcement learning algorithm will be

$$
\begin{aligned}
\tilde{V}_{n}^{k+1}(i)= & f(i) \breve{V}_{n}^{k}(i), \\
\tilde{V}_{t}^{k+1}(i)= & \left(1-a(k) I\left\{\breve{X}_{t}^{k+1}=i\right\}\right) \tilde{V}_{t}^{k}(i) \\
& +a(k) I\left\{\breve{X}_{t}^{k+1}=i\right\}\left(\frac{p\left(\breve{X}_{t+1}^{k+1}, Y_{t+1} \mid i\right)}{q\left(\breve{X}_{t+1}^{k+1} \mid i\right)}\right) \\
& \tilde{V}_{t+1}^{k+1}\left(\breve{X}_{t+1}^{k+1}\right), 0 \leq t<n, \\
\check{V}_{N}^{k+1}(i)= & 1, \\
\check{V}_{t}^{k+1}(i)= & \left(1-a(k) I\left\{\breve{X}_{t}^{k+1}=i\right\}\right) \check{V}_{t}^{k}(i) \\
& +a(k) I\left\{\breve{X}_{t}^{k+1}=i\right\}\left(\frac{p\left(\breve{X}_{t+1}^{k+1}, Y_{t+1} \mid i\right)}{q\left(\breve{X}_{t+1}^{k+1} \mid i\right)}\right) \\
& \check{V}_{t+1}^{k+1}\left(\breve{X}_{t+1}^{k+1}\right), t<N .
\end{aligned}
$$

As in Theorem 2, we can argue that a.s., $\check{V}_{n} \rightarrow \hat{V}_{n}$ a.s. Hence,

$$
\begin{aligned}
& \pi_{0} \tilde{V}_{n}^{k} \rightarrow v_{n}\left(f \hat{V}_{n}\right), \\
& \pi_{0} \check{V}_{0}^{k} \rightarrow v_{N} \mathbf{1} .
\end{aligned}
$$

This allows us to estimate the desired quantity $\frac{v_{n}\left(f \hat{V}_{n}\right)}{v_{N} \mathbf{1}}$. Note that $n=0$ corresponds to a 'stochastic observer', estimating the initial state based on observations.

Remark The foregoing underscores the difficulty in estimating cumulative performance measures of the form $E\left[\sum_{m=0}^{N} f\left(X_{m}\right) \mid Y_{m}, m \leq N\right]$, because this involves one filtering problem for estimating

$$
E\left[f\left(X_{N}\right) \mid Y_{m}, m \leq N\right]
$$

and $N$ smoothing problems for estimating

$$
E\left[f\left(X_{k}\right) \mid Y_{m}, m \leq N\right], 0 \leq k<N .
$$

An alternative is to augment the state space by introducing the new state variable $Z_{n}=\sum_{m=0}^{n} f\left(X_{m}\right), 0 \leq m \leq N$, and estimate $E\left[Z_{N} \mid Y_{m}, m \leq N\right]$ for the augmented Markov chain $\left(X_{n}, Z_{n}\right), n \geq 0$. The state space of $\left\{Z_{n}\right\}$, however, can get unwieldy. Thus this approach is not without its problems.

2. Continuous state/observation space: This extension is straightforward if the transition kernel $p\left(d x, d y \mid x^{\prime}\right)$ satisfies the following:

(a) $p\left(d x, d y \mid x^{\prime}\right)$ is of the form $\varphi_{y}\left(d x \mid x^{\prime}\right) \lambda(d y)$ for some positive measure $\lambda$ on $\mathcal{O}$ and a 'partial density' w.r.t. $\lambda$ given by $\varphi_{y}\left(d x \mid x^{\prime}\right)$,

(b) $\varphi_{y}\left(d x \mid x^{\prime}\right)$ above is absolutely continuous w.r.t. some transition kernel $q\left(d x \mid x^{\prime}\right)$ with the Radon-Nikodym derivative

$$
\Lambda\left(x, y \mid x^{\prime}\right):=\frac{d \varphi_{y}\left(d x \mid x^{\prime}\right)}{d q\left(d x \mid x^{\prime}\right)}
$$

being bounded and continuous in all variables.

Then we replace the ratio

$$
\frac{p\left(\breve{X}_{n}^{k}, Y_{n+1} \mid x\right)}{q\left(\breve{X}_{n}^{k} \mid x\right)}
$$

by $\Lambda\left(\breve{X}_{n}^{k}, Y_{n+1} \mid x\right)$.

The rest is routine, though there are practical considerations that mandate further tweaks. We shall address some of these in the context of a specific example later. Note that if the state and/or observation space are/is noncompact, one requires additional moment hypotheses on the iterates in order to ensure their a.s. convergence.

We end this section with a remark on a computational issue. Since the matrices $P(y)$ are sub-stochastic, the quantities $v_{n}(f), v_{n}(\mathbf{1})$ become very small very fast. However, we are interested in the ratio thereof, so they can be sporadically re-initialized by multiplying them by a common scalar in order to bring their values to a prescribed dynamic range. We have done so in the numerical experiments reported later. 


\section{The EM algorithm}

Consider the HMM given by the (state, observation) processes $\left(X_{n}, Y_{n}\right), n \geq 0$, taking values in a finite product space $\mathcal{S} \times \mathcal{O}$, such that

$$
P\left(X_{n+1}=i, Y_{n+1}=j \mid X_{m}, Y_{m}, m \leq n\right)=p_{\theta^{*}}\left(i, j \mid X_{n}\right)
$$

for a prescribed transition probability function $p_{\theta^{*}}(\cdot, \cdot \cdot \cdot)$. The latter is known to belong to a parametrized family $\left\{p_{\theta}(\cdot, \cdot \cdot \cdot): \theta \in \Theta\right\}$ for a compact parameter set $\Theta$ that contains the true parameter $\theta^{*}$. For simplicity of notation, we take $\Theta$ to be a closed bounded interval in $\mathcal{R}$, though what follows extends easily to closed and bounded parameter sets in $\mathcal{R}^{d}, d>1$. Let $\mathcal{F}_{n}:=\sigma\left(Y_{i}, i \leq n\right), n \geq 0$. We pick a $\hat{\theta}_{0}$ as our initial guess for $\theta^{*}$. The EM algorithm [13], the standard scheme for maximum likelihood estimation with partial data, is as follows when adapted to our context. It alternates between

1. (E step:) Estimate

$$
\Lambda\left(\theta, \theta^{\prime}\right):=E_{\theta}\left[\sum_{m=0}^{N-1} \log p_{\theta^{\prime}}\left(X_{m+1}, Y_{m+1} \mid X_{m}\right) \mid \mathcal{F}_{N}\right]
$$

for $\theta=\hat{\theta}_{n}$ based on observations $Y_{m}, 1 \leq m \leq N$, and

2. (M step:) Find $\hat{\theta}_{n+1}:=\operatorname{argmax}\left(\Lambda\left(\hat{\theta}_{n}, \cdot\right)\right)$

till convergence.

We begin with a stochastic approximation scheme for the first, or ' $E$ ', step here. For this, a 'stochastic approximation' or smoother based on 'reinforcement learning' in the spirit of the preceding section is given as follows. We fix a realization $Y_{m}=y_{m}, 1 \leq m \leq N$, of the observation process. This features as a fixed parameter in the following scheme. Let $Q:=[[q(j \mid i)]]_{i, j \in \mathcal{S}}$ be an irreducible stochastic matrix such that $\max _{y} p(j, y \mid i)>0 \Longrightarrow q(j \mid i)>0$. Consider a Markov chain $\left\{\tilde{X}_{n}\right\}$ with transition matrix $Q$ and initial distribution $\pi_{0}$. The law of this chain will be our 'importance sampling' measure. Simulate independent runs $\left\{\tilde{X}_{m}^{k}, 0 \leq m \leq N\right\}, k \geq 1$, of the chain with initial distribution $\pi_{0}$. The reinforcement learning smoother is, for $k=1,2, \cdots$, the following.

1. STEP 1: This step evaluates the common normalizing factor.

$$
\begin{aligned}
\hat{V}_{n}^{k+1}(i)= & \left(1-a(k) I\left\{\tilde{X}_{n}^{k+1}=i\right\}\right) \\
& \hat{V}_{n}^{k}(i)+a(k) I\left\{\tilde{X}_{n}^{k+1}=i\right\} \\
& \times\left(\frac{p_{\hat{\theta}_{k}}\left(\tilde{X}_{n+1}^{k+1}, y_{n+1} \mid i\right)}{q\left(\tilde{X}_{n+1}^{k+1} \mid i\right)}\right) \times\left(\hat{V}_{n+1}^{k+1}\left(\tilde{X}_{n+1}^{k+1}\right)\right), \\
& n<N,
\end{aligned}
$$

with terminal condition $\hat{V}_{N}^{k+1}(i)=1$.

2. STEP 2: This step evaluates unnormalized conditional expectation of the log-likelihood at parameter $\theta=$ the current estimate of $\theta^{*}$.

$$
\begin{aligned}
\tilde{V}_{n}^{k+1}(i)= & \left(1-a(k) I\left\{\tilde{X}_{n}^{k+1}=i\right\}\right) \\
& \tilde{V}_{n}^{k}(i)+a(k) I\left\{\tilde{X}_{n}^{k+1}=i\right\}\left(\frac{p_{\hat{\theta}_{k}}\left(\tilde{X}_{n+1}^{k+1}, y_{n+1} \mid i\right)}{q\left(\tilde{X}_{n+1}^{k+1} \mid i\right)}\right) \\
& \times\left(\log p_{\hat{\theta}_{k}}\left(\tilde{X}_{n+1}^{k+1}, y_{n+1} \mid i\right)\right. \\
& \left.\hat{V}_{n+1}^{k}\left(\tilde{X}_{n+1}^{k+1}\right)+\tilde{V}_{n+1}^{k+1}\left(\tilde{X}_{n+1}^{k+1}\right)\right), n<N
\end{aligned}
$$

with terminal condition $\tilde{V}_{N}^{k+1}(i)=0$.

3. STEP 3: This step evaluates unnormalized conditional expectation of the log-likelihood at parameter $\theta=\mathrm{a}$ perturbation of the current estimate of $\theta^{*}$.

$$
\begin{aligned}
\breve{V}_{n}^{k+1}(i)= & \left(1-a(k) I\left\{\tilde{X}_{n}^{k+1}=i\right\}\right) \\
& \breve{V}_{n}^{k}(i)+a(k) I\left\{\breve{X}_{n}^{k+1}=i\right\}\left(\frac{p_{\hat{\theta}_{k}}\left(\tilde{X}_{n+1}^{k+1}, y_{n+1} \mid i\right)}{q\left(\tilde{X}_{n+1}^{k+1} \mid i\right)}\right) \\
& \times\left(\log p_{\hat{\theta}_{k}+\delta}\left(\tilde{X}_{n+1}^{k+1}, y_{n+1} \mid i\right)\right. \\
& \left.\hat{V}_{n+1}^{k}\left(\tilde{X}_{n+1}^{k+1}\right)+\breve{V}_{n+1}^{k+1}\left(\tilde{X}_{n+1}^{k+1}\right)\right), n<N
\end{aligned}
$$

with terminal condition $\breve{V}_{N}^{k+1}(i)=0$.

Here, $\{a(k)\}$ is a positive sequence of step-sizes satisfying the standard conditions $\sum_{k} a(k)=\infty, \sum_{k} a(k)^{2}<\infty$. As before, the iteration (10) is nothing but a reinforcement learning scheme for 'policy evaluation' of the constant policy (here, uncontrolled) Markov chain $\left\{\tilde{X}_{n}\right\}$ with transition matrix $Q$ and the 'finite horizon cost'

$$
E\left[\prod_{m=1}^{N}\left(\frac{p_{\hat{\theta}_{k}}\left(\tilde{X}_{m+1}, y_{m+1} \mid \tilde{X}_{m}\right)}{q\left(\tilde{X}_{m+1} \mid \tilde{X}_{m}\right)}\right)\right] .
$$

That is, it is a stochastic approximation scheme for solving the corresponding 'dynamic programming equation' given by $V(\cdot, N) \equiv 1$ and for $m<N$,

$$
\begin{aligned}
\hat{V}(i, m) & =\sum_{j \in \mathcal{S}} q(j \mid i)\left(\frac{p_{\hat{\theta}_{k}}\left(j, y_{m+1} \mid i\right)}{q(j \mid i)}\right) \hat{V}(j, m+1) \\
& =\sum_{j \in \mathcal{S}} p_{\hat{\theta}_{k}}\left(j, y_{m+1} \mid i\right) \hat{V}(j, m+1) .
\end{aligned}
$$

The second expression on the right of (13) shows that

$$
\hat{V}(i, 0)=P\left(Y_{m}=y_{m}, 1 \leq m \leq N \mid X_{0}=i\right)
$$


for the Markov chain $\left\{X_{m}\right\}$ with associated observation process $\left\{Y_{m}\right\}$, governed by transition probabilities $p_{\hat{\theta}_{m}}(\cdot, \cdot \mid \cdot), 0 \leq m<N$. Note that $\sum_{i} \pi_{0}(i) \hat{V}(i, 0)$ is also the normalizing factor for the passage from normalized to unnormalized filter or smoother. On the other hand, (11) is a reinforcement learning scheme for evaluating

$$
\begin{aligned}
& E\left[\sum_{m=0}^{N-1}\left(\frac{p_{\hat{\theta}_{k}}\left(\tilde{X}_{m+1}, y_{m+1} \mid \tilde{X}_{m}\right)}{q\left(\tilde{X}_{m+1} \mid \tilde{X}_{m}\right)}\right)\right. \\
& \left.\log p_{\hat{\theta}_{k}}\left(\tilde{X}_{m+1}, y_{m+1} \mid \tilde{X}_{m}\right) \hat{V}_{n+1}^{k}\left(\tilde{X}_{n+1}^{k+1}\right)\right]
\end{aligned}
$$

for a Markov chain $\left\{\tilde{X}_{m}\right\}$ governed by the transition matrix $Q$. To see this, note that (14) equals $\sum_{i} \pi_{0}(i) \tilde{V}(i, 0)$, where $\tilde{V}(i, m), i \in \mathcal{S}, 0 \leq m \leq N$, is given by the 'dynamic programming equation'

$$
\begin{aligned}
\tilde{V}(i, m)= & \sum_{j} q(j \mid i)\left(\frac{p_{\hat{\theta}_{k}}\left(j, y_{m+1} \mid i\right)}{q(j \mid i)}\right) \\
& \log \left(p_{\hat{\theta}_{k}}\left(j, y_{m+1} \mid i\right)\right) \hat{V}_{n+1}^{k}(j) \\
& \quad+\sum_{j} q(j \mid i)\left(\frac{p_{\hat{\theta}_{k}}\left(j, y_{m+1} \mid i\right)}{q(j \mid i)}\right) \tilde{V}(j, m+1) \\
= & \sum_{j} p_{\hat{\theta}_{k}}\left(j, y_{m+1} \mid i\right) \log \left(p_{\hat{\theta}_{k}}\left(j, y_{m+1} \mid i\right)\right) \hat{V}_{n+1}^{k}(j) \\
& +\sum_{j} p_{\hat{\theta}_{k}}\left(j, y_{m+1} \mid i\right) \tilde{V}(j, m+1) .
\end{aligned}
$$

Iterating the second equality, it follows that $\sum_{i} \pi_{0}(i) \tilde{V}(i, 0)$ equals the finite horizon cost

$$
E\left[\left(\sum_{m=0}^{N-1} \log p_{\hat{\theta}_{k}}\left(X_{m+1}, y_{m+1} \mid X_{m}\right)\right) I\left\{Y_{m}=y_{m} \forall m \leq N\right\}\right]
$$

for the Markov chain $\left\{X_{m}\right\}$ with associated observation process $\left\{Y_{m}\right\}$, governed by transition probabilities $p_{\hat{\theta}_{k}}(\cdot, \cdot \mid \cdot)$. In this case, (11) is precisely the asynchronous stochastic approximation scheme to solve (15) or ipso facto, evaluate (16). In other words, it is a reinforcement learning scheme for approximate dynamic programming for this instance.

Likewise, (12) is a reinforcement learning scheme for exactly the same computation as that of (11), except that the 'running cost' $\log p_{\hat{\theta}_{k}}\left(X_{m+1}, y_{m+1} \mid X_{m}\right)$ in (16) is now replaced by $\log p_{\hat{\theta}_{k}+\delta}\left(X_{m+1}, y_{m+1} \mid X_{m}\right)$.

For the ' $M$ ' step, let

$$
F_{k}:=\frac{\pi_{0} \tilde{V}_{0}}{\pi_{0} \hat{V}_{0}}, F_{k}^{\prime}:=\frac{\pi_{0} \breve{V}_{0}}{\pi_{0} \hat{V}_{0}} .
$$

Consider the approximate gradient ascent (Kiefer-Wolfowitz scheme)

$$
\hat{\theta}_{k+1}=\hat{\theta}_{k}+b(k)\left(\frac{F_{k}^{\prime}-F_{k}}{\delta}\right),
$$

with $b(k)=\mathrm{O}(a(k))$. The latter condition ensures that this is a two-time-scale stochastic approximation (Chapter 6 of [12]) and the standard analysis of this class of schemes described in [12] applies. In particular, we can analyse the fast time scale iteration, i.e., the ' $E$ ' step, assuming that $\theta$, evaluated on the slower time scale, is frozen $(\approx$ 'quasistatic') and derive its limit. This yields estimates for the corresponding value functions. In turn, the slow time scale iteration for the parameter estimates $\hat{\theta}_{k}$ see the fast time scale computation of the ' $E$ ' step as quasi-equilibrated and therefore the term multiplying $b(k)$ above tracks the finite difference approximation to the desired gradient. Thus (17) is essentially an approximate gradient ascent for conditional expectation of the log-likelihood given observations, as desired by the EM methodology. It converges to a small neighborhood of a local maximum by a standard argument, see, e.g., Theorem 1, p. 339, [14].

\section{Numerical experiments}

\subsection{Queueing example}

Consider a queueing system

$$
Q_{n+1}=\left(Q_{n}-D_{n} I\left\{X_{n}>0\right\}+\xi_{n+1}\right) \bigwedge 100 .
$$

Here, given $0<b<<a<1$,

- $D_{n}$ (the departure process) is i.i.d., $D_{n}=1$ with probability $\mu_{n} \in\{a, b\}, n \geq 0$, and 0 otherwise,

- $\xi_{n}$ (the arrival process) is i.i.d., $\xi_{n}=1$ with probability $b<\lambda<a$ and 0 otherwise,

- $\left\{\mu_{n}\right\}$ (the service rate) is an $\{a, b\}$-valued Markov chain with two states: 'working' when $\mu_{n}=a$ and 'faulty' where $\mu_{n}=b$.

The chain $\left\{\mu_{n}\right\}$ transits from $a$ to $b$ with probability 0.01 and stays in $a$ with probability 0.99 . It moves from $b$ to $a$ with probability 0.9 and stays in $b$ with probability 0.1 . Denote by $p_{2}(\cdot \mid \cdot)$ the transition probabilities of $\left\{\mu_{n}\right\}$. We have limited the maximum queue size to 100 , i.e., assume a finite buffer of size 100. In case of buffer overflow, the extra arrivals are assumed lost.

In this example the observed process is the queue length $\left\{Q_{n}\right\}$ and the state process is $\left\{\mu_{n}, Q_{n}\right\}$. Comparing with our earlier notation, the correspondence is:

$$
X_{n} \longleftrightarrow\left\{\mu_{n}, Q_{n}\right\}, Y_{n} \longleftrightarrow Q_{n} .
$$

Thus $\quad X_{n} \in\{a, b\} \times\{1, \ldots, 100\}:=\mathcal{S}$, $Y_{n} \in\{1, \ldots, 100\}:=\mathcal{O}$. The transition probability function is: 


$$
\begin{aligned}
p\left(\left(u^{\prime}, i^{\prime}\right), \tilde{i} \mid(u, i)\right): & =P\left(X_{t+1}=\left(u^{\prime}, i^{\prime}\right), Y_{n+1}=\tilde{i} \mid X_{n}=(u, i)\right) \\
& =p_{1}\left(i^{\prime} \mid i, u\right) p_{2}\left(u^{\prime} \mid u\right) \delta_{i^{\prime}} \dot{i},
\end{aligned}
$$

where, for $0<i<100$,

$$
\begin{aligned}
p_{1}\left(i^{\prime} \mid i, u\right) & =\lambda(1-u), & & \text { for } i^{\prime}=i+1, \\
& =(1-\lambda) u, & & \text { for } i^{\prime}=i-1, \\
& =u \lambda+(1-u)(1-\lambda), & & \text { for } i^{\prime}=i, \\
& =0, & & \text { otherwise, }
\end{aligned}
$$

for $i=0$,

$$
\begin{aligned}
& p_{1}\left(i^{\prime} \mid i, u\right)=\lambda(1-u), \quad \text { for } i^{\prime}=i+1, \\
& =u+(1-u)(1-\lambda), \quad \text { for } i^{\prime}=i, \\
& =0, \quad \text { otherwise, }
\end{aligned}
$$

and for $i=100$,

$$
\begin{array}{rlrl}
p_{1}\left(i^{\prime} \mid i, u\right) & =(1-\lambda) u, & \text { for } i^{\prime}=i-1, \\
& =u \lambda+(1-u), & \text { for } i^{\prime}=i, \\
& =0, & & \text { otherwise. }
\end{array}
$$

In the following sample plots, the various parameter values are as follows.

- The Markov chain is evaluated for $N$ time steps. Here, $N=100$.

- $a=0.8$.

- $b=0.1$.

- $\lambda=0.5$.

- For transition matrix $Q$, the transition probabilities are as follows:

- probability to go from $a$ to $b=0.1$ and to stay at $a=0.9$,

- probability to go from $b$ to $a=0.7$ and to stay at $b=$ 0.3 .

It is seen that the naive importance sampling (i.e., fixed $q(\cdot \cdot \cdot)$ ) based reinforcement learning scheme achieves significant variance reduction. We have shown only a sample plot here. It was observed that the variance reduction improved with $N$, making the additional computational overhead worthwhile. As for adaptive importance sampling, we found that if used in isolation with initial guess for value function way off the mark, the initial transients were very bad. This suggested using naive importance sampling for an initial period and then switching to adaptive importance sampling. The reduction in variance was impressive, albeit at the expense of a significant increase in simulation budget ${ }^{1}$ and overall computational cost. Because of this, we did not use adaptive importance sampling for our numerical experiments for EM below. Nevertheless, it is a possibility worth keeping in mind.

\footnotetext{
${ }^{1}$ Double, because the importance sampling measures for two "value functions' corresponding to terminal condition $f$ and $\mathbf{1}$ differ unlike in the non-adaptive case, where it was fixed as the common $q(\cdot \mid \cdot)$.
}

We now turn to the EM algorithm. In the following sample plots, the various parameter values are:

- The Markov chain is evaluated for $\mathrm{N}$ time steps. Here, $N=20$.

- $a=0.8$.

- $b=0.1$.

- $\lambda=0.5$.

- The actual transition probabilities of the service rate chain are

- Probability to go from a to $\mathrm{b}=0.4$ and to stay in $\mathrm{a}=$ 0.6 ,

- Probability to go from $\mathrm{b}$ to $\mathrm{a}=0.7$ and to stay in $\mathrm{b}=$ 0.3 .

Our aim is to estimate the probability of transition from $a$ to $b$.

- The transition probabilities used in the importance sampling measure

- Probability to go from a to $\mathrm{b}=0.3$ and to stay in $\mathrm{a}=$ 0.7 ,

- Probability to go from $\mathrm{b}$ to $\mathrm{a}=0.8$ and to stay in $\mathrm{b}=$ 0.2 .

- $\delta=0.01$.

- $a(n)=\frac{1}{1+\left\lceil\frac{n}{M}\right\rceil}$ for $M=1000$.

- $b(n)=\frac{1}{2+\left\lceil\frac{m \log m}{N^{\prime}}\right\rceil}$ for $n=m M^{\prime}, m=0,1, \ldots, \quad$ and 0 otherwise, where $M^{\prime}=500$ and $N^{\prime}=10$.

As seen in figure 1, the scheme showed consistent convergence, though to a value dependent on the observation trace. The accuracy of the estimate tended to improve for higher $N$ as expected. The EM algorithm does not guarantee convergence to the global maxima of the partial likelihood function. To counter this we simulated the same experiment with the same parameters but with different initial guesses as reported above. If all the plots for a particular case converge to the same value and the initial guesses are well spread out in the domain, we take the point as our estimate. If the simulations with different initial guesses converge to different points in the domain, it means that they converge to different local maxima and we can in principle choose the best (in terms of having the highest estimated likelihood) among them as the estimate. We did not encounter this situation in our examples.

\section{A continuous state space example}

Consider the dynamics

$$
\begin{aligned}
X_{n+1} & =\frac{1}{2} X_{n}+\frac{\theta^{*}}{1+\left\|X_{n}\right\|^{2}}+W_{n+1}, \\
Y_{n+1} & =X_{n}+\xi_{n+1},
\end{aligned}
$$

where $\left\{W_{n}, \xi_{n}\right\}$ are IID $N(0,1)$. 

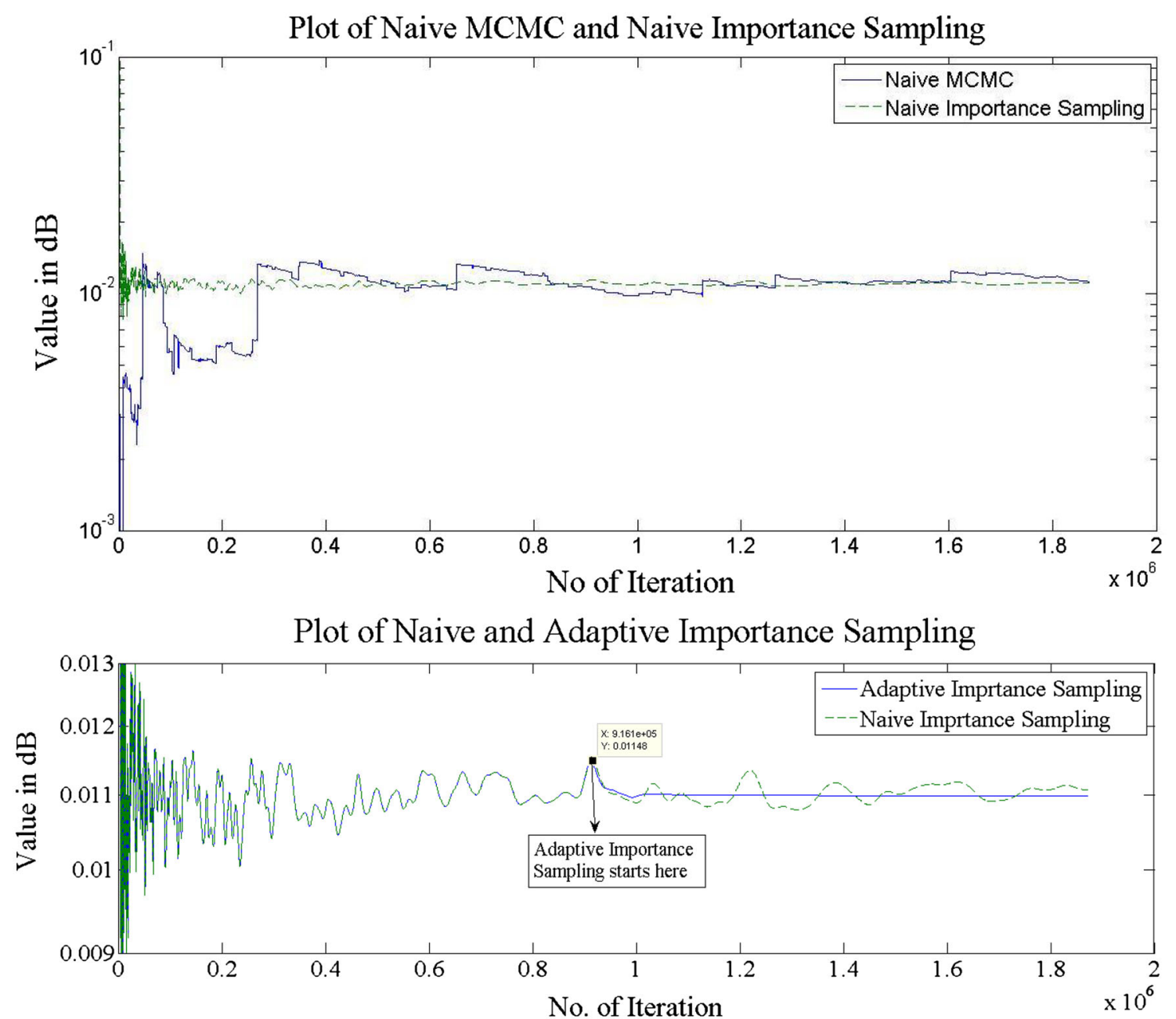

Figure 1. $N=100$ : Plot of naive MCMC and naive importance sampling versus no. of iterations and plot of naive importance sampling and adaptive importance sampling versus no. of iterations.

Here $X_{n}$ is the state process and $Y_{n}$ are the observations. As $X_{n}$ and $Y_{n}$ can take any value on the real line, the state space and the observation both are of continuous nature. $\theta^{*}$ is the parameter of interest which needs to be estimated. The Markov chain is run for $n=1$ to $n=N$.

The sample log-likelihood for EM at iterate $k$ will be proportional to

$$
\begin{aligned}
\Lambda^{k}(\theta) & :=-E_{\hat{\theta}^{k}}\left[\frac { 1 } { 2 } \sum _ { m = 0 } ^ { N - 1 } \left(\left(X_{m+1}^{k}-\frac{1}{2} X_{m}^{k}-\frac{\theta}{1+\left\|X_{m}^{k}\right\|^{2}}\right)^{2}\right.\right. \\
& \left.\left.+\left(Y_{m+1}^{k}-X_{m}^{k}\right)^{2}\right) \mid Y_{m}, m \leq N\right] .
\end{aligned}
$$

Set

$$
\begin{aligned}
& \log \left(p_{\theta^{k}}(X, Y \mid x)\right)=-\frac{1}{2}\left(X-\frac{x}{2}-\frac{\theta}{1+\|x\|^{2}}\right)^{2} \\
& -\frac{1}{2}(Y-x)^{2}+C
\end{aligned}
$$

for a constant $C$ and

$$
\begin{aligned}
& \frac{\partial}{\partial \theta} \log \left(p_{\theta^{k}}(X, Y \mid x)\right)=\left(X-\frac{x}{2}-\frac{\theta}{1+\|x(m)\|^{2}}\right) \\
& \times\left(\frac{1}{1+\|x(m)\|^{2}}\right) .
\end{aligned}
$$

The reinforcement learning scheme is: for $k=1,2, \ldots$, do

1. STEP 1: This step evaluates the common normalizing factor.

$$
\begin{aligned}
\hat{V}_{N}^{k+1}(i)= & 1, \\
\hat{V}_{n}^{k+1}(i)= & \left(1-a(k) I\left\{\tilde{X}_{n}^{k+1}=i\right\}\right) \\
& \check{V}_{n}^{k}(i)+a(k) I\left\{\tilde{X}_{n}^{k+1}=i\right\} \\
& \times\left(\frac{p_{\hat{\theta}^{k}}\left(\tilde{X}_{n+1}^{k+1}, y_{n+1} \mid i\right)}{q\left(\tilde{X}_{n+1}^{k+1} \mid i\right)}\right)\left(\hat{V}_{n+1}^{k+1}\left(\tilde{X}_{n+1}^{k+1}\right)\right), n<N .
\end{aligned}
$$


2. STEP 2: This step evaluates unnormalized conditional expectation of the gradient of the log-likelihood at parameter $\theta=$ the current estimate of $\theta^{*}$.

$$
\begin{aligned}
& \tilde{V}_{N}^{k+1}(i)=0 \\
& \tilde{V}_{n}^{k+1}(i)=\left(1-a(k) I\left\{\tilde{X}_{n}^{k+1}=i\right\}\right) \\
& \tilde{V}_{n}^{k}(i)+a(k) I\left\{\tilde{X}_{n}^{k+1}=i\right\} \\
& \times\left(\frac{p_{\hat{\theta}^{k}}\left(\tilde{X}_{n+1}^{k+1}, y_{n+1} \mid i\right)}{q\left(\tilde{X}_{n+1}^{k+1} \mid i\right)}\right) \\
& \left(\frac{\partial}{\partial \theta}\left[\log p_{\hat{\theta}^{k}}\left(\tilde{X}_{n+1}^{k+1}, y_{n+1} \mid i\right)\right] \hat{V}_{n+1}^{k+1}\left(\tilde{X}_{n+1}^{k+1}\right)\right. \\
& \left.+\tilde{V}_{n+1}^{k+1}\left(\tilde{X}_{n+1}^{k+1}\right)\right), n<N .
\end{aligned}
$$

For the 'M' step, let

$$
F^{k}:=\frac{\pi_{0} \tilde{V}_{0}}{\pi_{0} \hat{V}_{0}}
$$

Consider the gradient ascent

$$
\hat{\theta}^{k+1}=\hat{\theta}^{k}+b(k) F^{k}
$$

with $b(k)=\mathrm{O}(a(k))$.

We benchmark this against a vanilla SMC scheme given as follows.
1. STEP 1: This step evaluates the common normalizing factor.

$$
\begin{aligned}
\hat{V}_{N}^{k+1}(i)= & 1, \\
\hat{V}_{n}^{k+1}(i)= & I\left\{\tilde{X}_{n}^{k+1}=i\right\}\left(\frac{p_{\hat{\theta}^{k}}\left(\tilde{X}_{n+1}^{k+1}, y_{n+1} \mid i\right)}{q\left(\tilde{X}_{n+1}^{k+1} \mid i\right)}\right) \\
& \times\left(\hat{V}_{n+1}^{k+1}\left(\tilde{X}_{n+1}^{k+1}\right)\right), n<N .
\end{aligned}
$$

2. STEP 2: This step evaluates unnormalized conditional expectation of the $\log$-likelihood at parameter $\theta=$ the current estimate of $\theta^{*}$.

$$
\begin{aligned}
& \tilde{V}_{N}^{k+1}(i)=0, \\
& \tilde{V}_{n}^{k+1}(i)=I\left\{\tilde{X}_{n}^{k+1}=i\right\}\left(\frac{p_{\hat{\theta}^{k}}\left(\tilde{X}_{n+1}^{k+1}, y_{n+1} \mid i\right)}{q\left(\tilde{X}_{n+1}^{k+1} \mid i\right)}\right) \\
& \quad \times\left(\frac{\partial}{\partial \theta}\left[\log p_{\hat{\theta}^{k}}\left(\tilde{X}_{n+1}^{k+1}, y_{n+1} \mid i\right)\right] \hat{V}_{n+1}^{k+1}\left(\tilde{X}_{n+1}^{k+1}\right)\right. \\
& \left.\quad+\tilde{V}_{n+1}^{k+1}\left(\tilde{X}_{n+1}^{k+1}\right)\right), n<N .
\end{aligned}
$$

For the 'M' step, let

$$
F^{k}:=\frac{\pi_{0} \sum_{j=0}^{k} \tilde{V}_{0}^{j}}{\pi_{0} \sum_{j=0}^{k} \hat{V}_{0}^{j}}
$$

Consider the gradient ascent

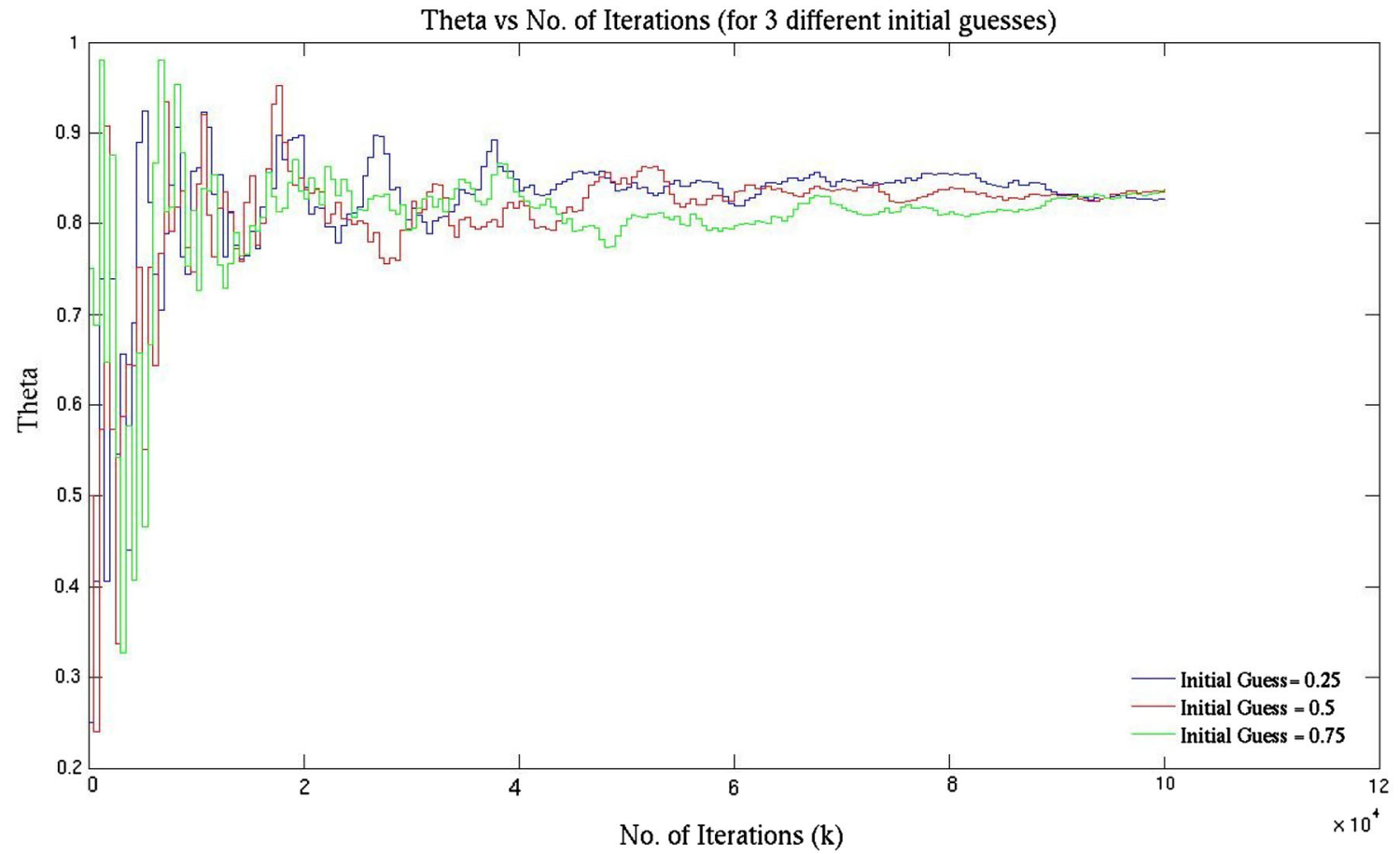

Figure 2. $N=20$ : Plot of theta versus no. of iterations for various initial guesses. 
Theta vs No. of Iterations for both the schemes

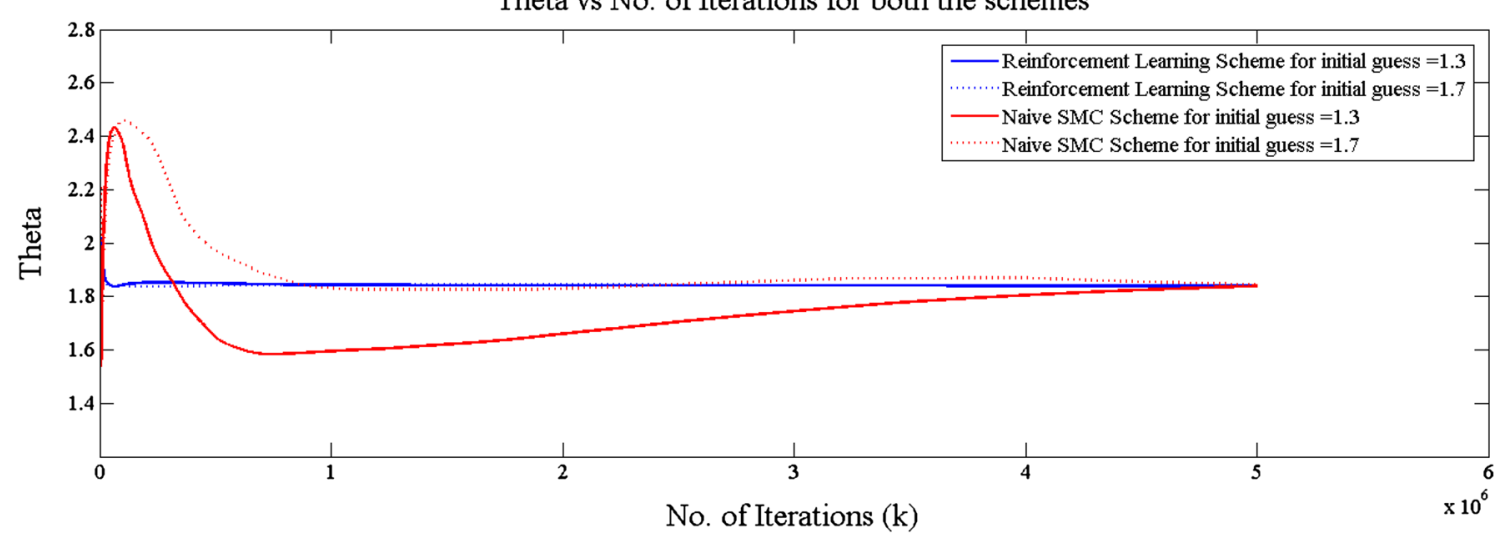

Figure 3. $N=20, K=100$ : Plot of theta versus no. of iterations for the two schemes for the two initial guesses.

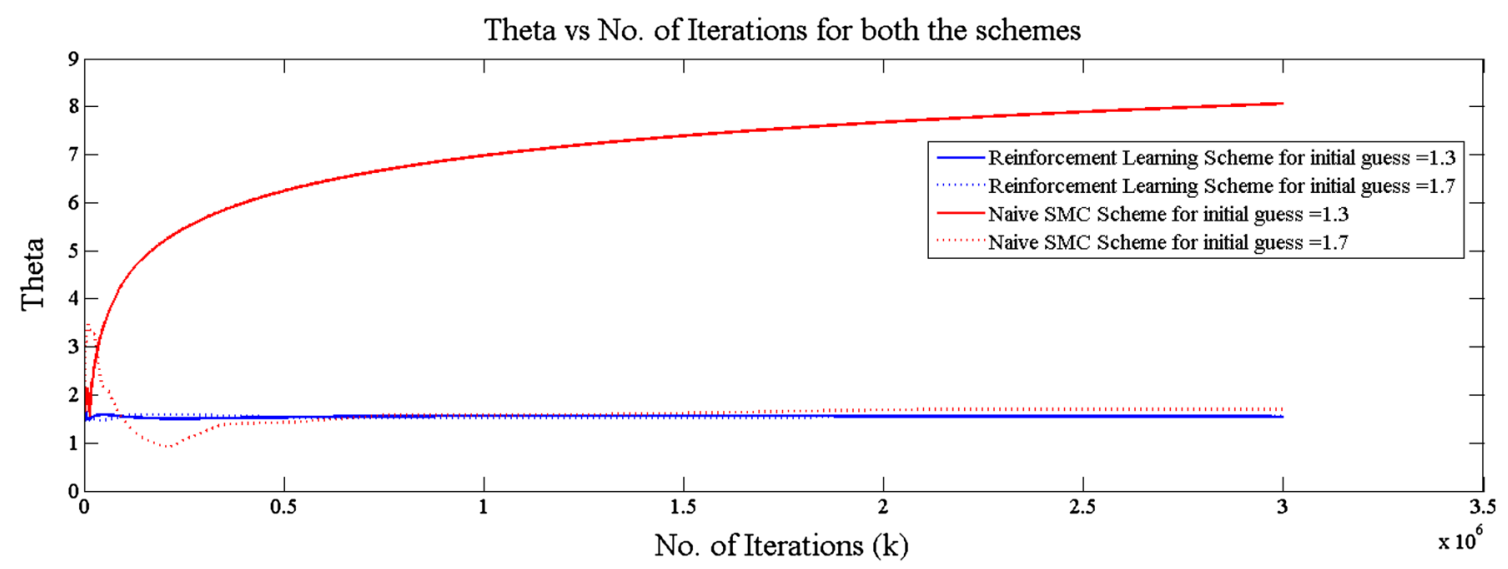

Figure 4. $N=30, K=80$ : Plot of theta versus no. of iterations for the two schemes and the two initial guesses.

$$
\hat{\theta}^{k+1}=\hat{\theta}^{k}+b(k) F^{k}
$$

with $b(k)=\mathrm{O}(a(k))$.

In our numerical experiments, we restrict $X_{n}$ and $Y_{n}$ in the range $(-5,5)$ as with high probability they do not exceed these bounds. We discretize this range into $M$ subregions. Each is represented by a point (node). From this we evaluate the transition probability matrix by sampling from the continuous density at the grid points and normalizing, and then execute the algorithm. $K$ is varied to make the grid as fine as possible without making the algorithm computationally prohibitively expensive.

In the following simulation results, we have set $\theta^{*}=1.4$. We evaluate both the schemes for two initial guesses of 1.3 and 1.7. We have used $K=60, K=80, K=100$ and $K=200$. As seen in figures 2, 3 and 4, both the schemes give consistent convergence. The reinforcement-learningbased scheme has considerably lower variance compared with the non-reinforcement learning scheme. As we increase $K$, it takes more time to converge as expected. Also, as we increase $N$, the non-reinforcement learning scheme may lead to numerical instability as seen in the results for $N=30$.

\section{Conclusion and future work}

We have proposed variants of SMC and an EM algorithm for HMMs wherein ideas from reinforcement learning for approximate dynamic programming have been incorporated to ensure variance reduction leading to a more graceful convergence. However, introducing these techniques also has its problems. While reinforcement learning inherits the advantages of MCMC and deterministic numerical schemes, it also inherits some of their disadvantages. In particular it inherits the curse of dimensionality from numerical schemes and slow convergence from Monte Carlo due to slow approach to stationarity caused by possible occurrence of quasi-invariant subsets of the state space. One popular approach to tackle this curse of dimensionality in reinforcement learning has been the use of function approximation. It would be interesting to see 
whether such approximation architectures can be used to advantage in the present set-up.

\section{References}

[1] Borkar V S, Jain A V 2014 Reinforcement learning, particle filters and the EM algorithm. In: Proceedings of the Workshop on Information Theory and Applications, San Diego, February

[2] Bain A and Crisan D 2009 Fundamentals of stochastic filtering. Berlin-Heidelberg: Springer Verlag

[3] Asmussen S and Glynn P W 2007 Stochastic simulation: algorithms and analysis. New York: Springer Verlag

[4] Borkar V S 2009 Reinforcement learning - a bridge between numerical methods and Monte Carlo. In: Sastry N S N, Rao T S S R K, Delampady M and Rajeev B (Eds.) Perspectives in mathematical sciences I: probability and statistics. Singapore: World Scientific, pp. 71-91

[5] Zakai M 1969 On the optimal filtering of diffusion processes. Zeitschrift für Wahrscheinlichkeitstheorie und Verwandte Gebiete 11(3): 230-243
[6] Borkar V S 1991 A remark on control of partially observed Markov chains. Annals of Operations Research 29(1): 429-438

[7] Borkar V S 1991 Topics in controlled Markov chains. Pitman Research Notes in Maths. No. 240. Harlow, UK: Longman Scientific and Technical

[8] Elliott R J, Aggoun L and Moore J B 1995 Hidden Markov models: estimation and control. New York: Springer Verlag

[9] Ahamed T P I, Borkar V S and Juneja S 2006 Adaptive importance sampling technique for Markov chains using stochastic approximation. Operations Research 54(3): 489-504

[10] Lindsten F and Schön T B 2013 Backward simulation methods for Monte Carlo statistical inference. In: Foundations and Trends in Machine Learning, vol. 6(1), Hanover, MA: NOW Publishers, pp. 1-143

[11] Cappé O, Moulines E and Rydén T 2005 Inference in hidden Markov models. New York: Springer Verlag

[12] Borkar V S 2008 Stochastic approximation: a dynamical systems viewpoint. New Delhi, India: Hindustan Book Agency and Cambridge, UK: Cambridge University Press

[13] Dempster A P, Laird N M and Rubin D B 1977 Maximum likelihood from incomplete data via the EM algorithm. Journal of the Royal Statistical Society Series B 39(1): 1-38

[14] Hirsch M W 1989 Convergent activation dynamics in continuous time networks. Neural Networks 2: 331-349 